\section{Commentary: Complex transposition: Preparing for success}

\author{
Arshid Mir, MD, ${ }^{\mathrm{a}}$ and Harold M. Burkhart, $\mathrm{MD}^{\mathrm{b}}$ \\ "By failing to prepare, you are preparing to fail." \\ —Benjamin Franklin
}

Complex transposition consisting of D-transposition of the great arteries (TGA), ventricular septal defect (VSD), and left ventricular outflow tract obstruction (LVOTO) has a heterogeneous variation in cardiac defects. A wide variability in the VSD location (perimembranous or nonperimembranous/remote), the etiology of LVOTO, which can be either subvalvular (discrete or tunnel-like) or valvar (hypoplastic or stenotic), as well as assorted coronary artery patterns can present surgical challenges to achieving the best repair. Several surgical options for this condition exist, with some patients being candidates for an early or delayed arterial switch operation (ASO) and others benefiting from a Rastelli or aortic translocation approach when an ASO operation is infeasible. Previously, due to difficulties in understanding the 3-dimensional (3D) spatial location of the VSD and the need to use anatomic imagination in preoperative planning, the surgical decision was often made in the operating room after inspection of the coronary artery anatomy together with the VSD location and its relationship to the outflow tract.

In this issue of the JTCVS Techniques, Contreras and colleagues $^{1}$ demonstrate how a combination of different imaging modalities was used to plan a successful surgical

From the ${ }^{\mathrm{a} S e c t i o n}$ of Pediatric Cardiology and ${ }^{\mathrm{b}}$ Division of Cardiovascular and Thoracic Surgery, University of Oklahoma Health Sciences Center, Oklahoma City, Okla.

Disclosures: The authors reported no conflicts of interest.

The Journal policy requires editors and reviewers to disclose conflicts of interest and to decline handling or reviewing manuscripts for which they may have a conflict of interest. The editors and reviewers of this article have no conflicts of interest.

Received for publication Feb 14, 2021; revisions received Feb 14, 2021; accepted for publication Feb 22, 2021; available ahead of print Feb 27, 2021.

Address for reprints: Harold M. Burkhart, MD, Division of Cardiovascular and Thoracic Surgery, University of Oklahoma Health Sciences Center, PO Box 26901, WP-2230, Oklahoma City, Okla 73105 (E-mail: Harold-burkhart@ouhsc. edu).

JTCVS Techniques 2021;7:206-7

2666-2507

Copyright (C 2021 The Authors. Published by Elsevier Inc. on behalf of The American Association for Thoracic Surgery. This is an open access article under the CC BY-NCND license (http://creativecommons.org/licenses/by-nc-nd/4.0/).

https://doi.org/10.1016/j.xjtc.2021.02.036

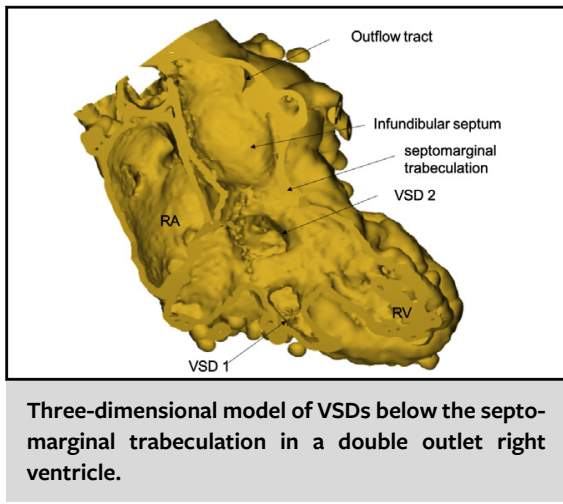

CENTRAL MESSAGE

Multimodal imaging with tailor-

made 3-dimensional printed

models are invaluable to the

preoperative planning process.

repair in a case of complex TGA. The 3D models allowed the exclusion of certain operations owing to concerns about LVOT size and coronary location. The authors acknowledge that these tailor-made models made it easier to understand the anatomy and propose a surgical solution involving VSD enlargement with a Rastelli intracardiac pathway while incorporating the pulmonary valve into the LVOT. The superb accompanying video reviewing the anatomic models and thought processes in preoperative planning represents an exemplary approach to these complex repairs.

Recent advances in cardiac magnetic resonance imaging and computed tomography with the use of 3D printing has helped appreciate key anatomic details such as location of a VSD in 3D spatial orientation rather than 2-dimensional (2D) echocardiographic images (Figure 1). The greatest limitation in reviewing 2D planar echo imaging is the difficulty appreciating the technical and surgical challenges that are not apparent before surgery. ${ }^{2,3}$ The tailored, en face models obtained from the right ventricular (RV) side are similar to what a surgeon sees during the operation. These models can clearly delineate the location of a VSD from the RV surface, the location of the infundibular septum, and the location of the nearest outflow tract. ${ }^{4}$ This can aid planning in terms of the need for VSD enlargement, the location of the intracardiac baffle, and the resultant RV size if the proposed baffle is adopted. ${ }^{5}$ Apart from $3 \mathrm{D}$ printing and 3D bespoke models, augmented virtual reality (ie, 


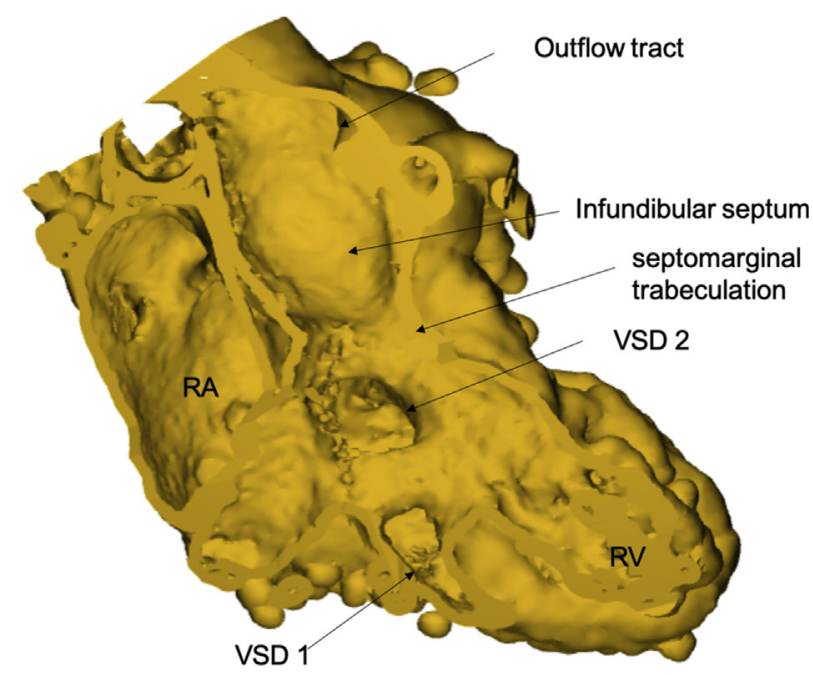

FIGURE 1. A 3D model with the anterior wall of the right ventricle removed showing 2 separate ventricular septal defects (VSDs) below the septomarginal trabeculation in a patient with a double-outlet right ventricle. $R A$, right atrium.

virtual holograms) from 3D models can aid the surgical team in inspecting and visualizing models with enhanced depth perception. ${ }^{6}$

The use of anatomic models in preoperative planning has the potential for improving outcomes in complex surgical patients. Knowing which surgical strategies are likely possible before the operation may decrease operative times while avoiding unnecessary surgical manipulations (eg, ventriculotomy) before definitive repair. Although unknown at this time, these may translate into improved neurodevelopmental outcomes and other factors contributing to morbidity (hospital stay, risk of reintervention) as well as lower health care costs.
The current limitations of these models are the inability to visualize the valvular and subvalvular apparatuses and the lack of dynamic assessment of the baffle. Hopefully through fusion hybrid imaging of echo and 3D printed models, these can be combined to aid patients in whom the proximity of the VSD baffle to the tricuspid valve presents uncertainties regarding atrioventricular valve function after baffle placement.

In conclusion, the report from Contreras and colleagues emphasizes and validates the use of rapid 3D prototyping in complex patients. We advocate the routine use of this technology in management of patients with complex intracardiac or extracardiac anatomy. Transparent 3D models can be used for surgical rehearsal so that outcomes can be accurately predicted and tailored for a patient's specific anatomy. Apart from preoperative surgical planning, the use of these models for surgical simulation and discussion with other caregivers and patients' families are additional benefits of this technology. When it comes to complex congenital anomalies, having all these advantages at our disposal allows us to prepare for success.

\section{References}

1. Contreras JR, Villemain O, Marini D, Dragulescu A, Yoo S-J, Barron DJ. Utility of a bespoke 3-dimensional printed model in complex tranposition. $J$ Thorac Cardiovasc Surg Tech. 2021;7:199-202.

2. Van Arsdell GS, Hussein N, Yoo SJ. Three-dimensional printing in congenital cardiac surgery—now and the future. J Thorac Cardiovasc Surg. 2020;160:515-9.

3. Vukicevic M, Mosadegh B, Min JK, Little SH. Cardiac 3D printing and its future directions. JACC Cardiovasc Imaging. 2017;10:171-84.

4. Yoo SJ, Van Arsdell GS. 3D printing in surgical management of double outlet right ventricle. Front Pediatr. 2018;5:289.

5. Forte MNV, Hussain T, Roest A, Gomez G, Jongbloed M, Simpson J, et al. Living the heart in three dimensions: applications of 3D printing in CHD. Cardiol Young. 2019;29:733-43.

6. Brun H, Bugge RAB, Suther LKR, Birkeland S, Kumar R, Pelanis E, et al. Mixed reality holograms for heart surgery planning: first user experience in congenital heart disease. Eur Heart J Cardiovasc Imaging. 2019;20:883-8. 